\title{
Foreword. The Lunar Crater Observation Sensing Satellite (LCROSS)
}

\author{
C.T. Russell • A. Colaprete
}

Published online: 5 May 2012

(C) Springer Science+Business Media B.V. 2012

The Moon is our nearest neighbor. It is natural that we should visit our neighbor frequently and we have. We have flown by the Moon, orbited the Moon and landed on the Moon. We find we have much in common with our neighbor. The Sun shines on the Moon with the same intensity as on Earth. The interior of the Moon, like the Earth, has a crust, mantle and core. The Moon's smaller size has produced some important differences, however. It lost its internal heat more rapidly than Earth and it has lost all but a very tiny amount of its atmosphere. Most importantly it has lost most of its original inventory of water, a resource that is important to human presence on this body, as well as most geologic processes with which we are familiar on Earth. Still it may possess its version of a polar cap.

Volatiles in vacuum environments migrate to the coldest spot exposed to them. Chemists have known this for centuries using 'cold traps' in their vacuum chambers. This same process acting on a planetary surface results in migration of some gases to the poles. One of these volatile gases is that most important resource, water. Several of our missions that have examined the polar regions of the Moon have returned evidence consistent with the presence of water, possibly buried but close to the surface. The launch of the lunar Reconnaissance Orbiter (LRO) opened up the possibility of investigating these possible deposits when the launch vehicle to be used for LRO had excess capacity not needed for the prime mission.

The Lunar Crater Observation Sensing Satellite (LCROSS) was selected in response to a request from NASA for ideas to use this "excess" launch capacity. Its goal was to determine the nature of materials within the permanently shadowed craters. It used the spent LRO Earth-lunar transfer rocket stage as a kinetic impactor that dove into a permanently shadowed region on 9 October 2009, throwing ejecta into sunlight where it could be seen. The impactor was guided to its target by a shepherding spacecraft which contained instruments to observe the impact. An accompanying observational campaign from Earth and

C.T. Russell ( $\bowtie)$

University of California, Los Angeles, Los Angeles, CA, USA

e-mail: ctrussel@igpp.ucla.edu

A. Colaprete

NASA Ames Research Center, Moffett Field, CA, USA 
lunar orbit complemented the observations by the shepherding satellite which itself became an impactor. This volume describes the LCROSS mission that enabled these observations.

The volume contains four articles, the first, by its lead scientist A. Colaprete and colleagues discusses the earlier observations that paved the way for the LCROSS investigation, the goals of the mission, its configuration, the design of the impact, contamination control, target selection and timing, instruments and the observational campaign. This overview is followed by a description of the payload development and its performance in flight by K. Ennico and colleagues. Next, W. Marshall and colleagues describe how the LCROSS impact craters were located. Finally, J.L. Heldmann and a cast of seemingly thousands describe the observation campaign and its strategies, implementation and lessons learned.

The success of a space mission such as LCROSS depends on the hard work and assistance of many dedicated individuals. In this instance not only were the members of the project team centered at NASA's Ames Research Center essential but also the scientists involved in the accompanying observing campaign and LRO project and LRO instruments leadership, with whom uniquely strong collaborations were formed. We thank all these individuals for their enabling contributions. We wish also to thank those who have facilitated the assembly of this description of the effort especially the authors who distilled the reports and documents that a mission produces into these highly readable papers. The editors benefited from the advice of an excellent set of referees who carefully read each paper and provided positive feedback to the authors that improved the papers. These referees included: Erik Asphaug, David Patrick O’Brien, Gordon Chin, Olivier Hainaut, Paul O. Hayne, Brendan Hermalyn, Mark Sykes,

Equally important has been the strong support expended by Lalitha Jaganathan and Harry Blom at Springer. At UCLA we were skillfully assisted by Marjorie Sowmendran who acted as the interface between authors, referees and the publisher. 